\title{
CHARACTERIZATION OF SCHWARTZ SPACES BY THEIR HOLOMORPHIC DUALS
}

\author{
STEN BJON AND MIKAEL LINDSTRÖM
}

(Communicated by Paul S. Muhly)

\begin{abstract}
Let $U$ be an open subset of a locally convex space $E$, and let $H_{c}(U, F)$ denote the vector space of holomorphic functions into a locally convex space $F$, endowed with continuous convergence. It is shown that if $F$ is a semiMontel space, then the bounded subsets of $H_{c}(U, F)$ are relatively compact. Further it is shown that $E$ is a Schwartz space iff the continuous convergence structure on the algebra $H(U)$ of scalar-valued holomorphic functions on $U$, coincides with local uniform convergence. Using this, an example of a nuclear Fréchet space $E$ is given, such that the locally convex topology associated with continuous convergence on $H(E)$ is strictly finer than the compact open topology. Thus, the behavior of the space $H_{c}(E)$ differs in this respect from that of its subspace $L_{c} E$ of linear forms and that of its superspace $C_{c}(E)$ of continuous functions.
\end{abstract}

Introduction. In [11] $\mathrm{H}$. Jarchow has proved that a locally convex space (lcs) $E$ is Schwartz if and only if continuous convergence and local uniform convergence coincide on the dual of $E$. It is natural to ask if there is a holomorphic analogue of this result: Is a space $E$ Schwartz if and only if continuous convergence and local uniform convergence coincide on a space $H(U)$ of holomorphic functions on some open subset $U$ of $E$ ? We give a positive answer to this question using a result, closely related to Jarchow's, for continuous $m$-homogeneous polynomials [5]. Further we prove that the space $H(U)$ has the Montel property (bounded sets are relatively compact) when endowed with continuous convergence.

For spaces of linear forms (on locally convex spaces) as well as for spaces of continuous functions (on e.g. completely regular spaces) it is known that the locally convex topology associated with continuous convergence is the compact-open topology (cf. [1 and 10]). Using the above holomorphic characterization of Schwartz spaces, we provide an example which shows that spaces of holomorphic functions behave quite differently: There exists a nuclear Fréchet space $E$, such that the locally convex topology associated with continuous convergence on $H(E)$ is strictly finer than the compact-open topology.

We recall some notation and definitions. All vector spaces in this paper are complex. A function $f: U \rightarrow F$ into a convergence vector space (cvs) $F$ is Gateauxholomorphic if the function $\lambda \mapsto l \circ f(x+\lambda h)$ is holomorphic in a neighborhood of zero for each $x \in U, h \in E$, and $l \in L F$. It is holomorphic if it is Gâteauxholomorphic and continuous. Let $H(U, F)$ be the vector space of holomorphic

Received by the editors January 20, 1987.

1980 Mathematics Subject Classification (1985 Revision). Primary 46G20; Secondary 46A12, 54A20.

Key words and phrases. Schwartz spaces, holomorphic function, convergence structure. 
functions $f: U \rightarrow F$. When endowed with continuous convergence [1] or with the associated equable convergence [8] (which coincides with local uniform convergence if $F$ is normed) it will be denoted by $H_{c}(U, F)$ and $H_{e}(U, F)$, respectively. A net $\left(f_{k}\right)_{k}$ converges to zero in $H_{c}(U, F)$ if and only if for each $x \in U$ and each net $\left(x_{l}\right)_{l}$, which converges to $x$, the net $\left(f_{k}\left(x_{l}\right)\right)_{k l}$ converges to zero in $F$. The net $\left(f_{k}\right)_{k}$ converges to zero in $H_{e}(U, F)$ if and only if for each $x \in U$ and each $\left(x_{l}\right)_{l}$, which converges to $x$, there is a filter $\mathcal{G}$ converging to zero in $F$, such that for each $G \in \mathcal{G}$ exists an $l_{0}$, such that $q_{G}\left(f_{k}\left(x_{l}\right)\right)$ converges to 0 uniformly on $\left\{x_{l}: l \geq l_{0}\right\}$. For cvs $E$ and $F$, let $P_{c}\left({ }^{m} E, F\right)$ and $P_{e}\left({ }^{m} E, F\right)$ be the subspaces of $H_{c}(U, F)$ and $H_{e}(U, F)$, respectively, consisting of all continuous $m$-homogeneous polynomials. A cvs $E$ is $L_{c}$-embedded (or $L_{e}$-embedded) if the mapping $j_{E}: E \rightarrow L_{c} L_{c} E$ (or $j_{E}: E \rightarrow$ $\left.L_{e} L_{e} E\right), j_{E}(x) l=l(x)$ into the second dual is an embedding [2]. All Hausdorff lcs are $L_{\alpha}$-embedded $(\alpha=c, e)$. Further $H_{c}(U, F)$ and $P_{c}\left({ }^{m} E, F\right), m \in \mathbf{N}$, are $L_{c^{-}}$ embedded spaces and $H_{e}(U, F)$ and $P_{e}\left({ }^{m} E, F\right), m \in \mathbf{N}$, are $L_{e}$-embedded spaces. A subset $B$ (or a filter $\mathcal{F}$ ) in a cvs $E$ is said to be bounded (or quasi-bounded [8]), if $\mathbf{V B} \rightarrow 0$ (or $\mathbf{V} \mathcal{F} \rightarrow 0$ ) in $E$, where $\mathbf{V}$ denotes the filter of neighborhoods of 0 in C. Note that a subset (or a filter) in $H(U, F)$ is bounded (or quasi-bounded) in $H_{c}(U, F)$ if and only if it is bounded (or quasi-bounded) in $H_{e}(U, F)$. Clearly all convergent filters in a cvs are quasi-bounded. The adherence $a(B)$ of a subset $B$ of $E$ is by definition the set $\{x \in E: \exists$ a filter $\mathcal{F} \rightarrow x$ with $B \in \mathcal{F}\}$. A subset $B$ of $E$ is said to be closed if $a(B)=B$, compact if every ultrafilter $\mathcal{U}$ with $B \in \mathcal{U}$ converges in $B$, and relatively compact if $a(B)$ is compact.

The Montel property. First we show that $H_{c}(U, F)$ has the Montel property, when $F$ is a semi-Montel space. The proof is based on Theorem 1, the proof of which can be found in [6] (cf. also [9]). If $X$ and $Y$ are convergence spaces, $C_{c}(X, Y)$ will denote the set of continuous functions from $X$ into $Y$, endowed with continuous convergence.

1. THEOREM. Let $X$ be a convergence space and $Y$ a uniform space, and let $B \subseteq C(X, Y)$. Then $B$ is relatively compact in $C_{c}(X, Y)$ if and only if it is equicontinuous and, for each $x \in X, B(x)$ is relatively compact in $Y$.

2. Proposition. Let $U$ be an open subset of an lcs $E$ and let $F$ be a semiMontel space. Then every bounded subset $B$ of $H(U, F)$ is relatively compact in $H_{c}(U, F)$.

Proof. Let $B$ be bounded in $H_{c}(U, F)$. Then, for each $x \in U$ and each circled convex 0 -neighborhood $V$ in $F$, there exists a 0 -neighborhood $W$ in $E$ with $x+W \subseteq U$ and $\varepsilon>0$, such that $\varepsilon B(x+W) \subseteq V$. Thus $B$ is pointwise bounded. Further, by Cauchy's inequality,

$$
\sup _{h \in W} q_{V}\left(1 / m ! \hat{d}^{m} f(x) h\right) \leq q_{V}(f(x+W)) \leq \varepsilon
$$

for $f \in B\left(q_{V}\right.$ denotes the gauge of $\left.V\right)$. Now

$$
q_{V}(f(y)-f(x)) \leq \sum_{m=1}^{\infty} q_{V}\left(\frac{1}{m !} \hat{d}^{m} f(x)(y-x)\right) \leq \varepsilon \sum_{m=1}^{\infty} \delta^{m}=\varepsilon \delta(1-\delta)^{-1}
$$

for arbitrary $y \in x+\delta W, \delta<1$. Hence $f(y)-f(x) \in \varepsilon \delta(1-\delta)^{-1} V$ for each $f \in B$, i.e. $B$ is equicontinuous. Since $F$ is semi-Montel, the set $B(x)(x \in U)$, is 
relatively compact in $F$. Theorem 1 yields the proposition, since $H(U, F)$ is closed in $C_{c}(U, F)$ according to Theorem 2.1 in [4].

REMARKS. (a) In $L_{c}$-embedded spaces, and thus also in $L_{e}$-embedded spaces, relatively compact subsets are bounded (cf. [3]). In the proof for Proposition 2 we showed that bounded subsets of $H_{c}(U, F)$ are equicontinuous and pointwise bounded. The converse statement is also true, as is easily seen.

(b) Let $U^{\text {lc }}$ denote the locally compact convergence space associated with $U$. Now $H_{H Y}(U, F) \cong H_{c}\left(U^{\mathrm{lc}}, F\right)$, where the space $H_{H Y}(U, F)$ of hypoanalytic functions is endowed with the compact-open topology (cf. [4]). Since, under the assumptions in (d) below, a subset of $H(U)$ is bounded in $H_{c}(U)$ if and only if it is bounded in $H_{c o}(U)$ (Proposition 3.5 in [4]), we obtain the well-known results [7]:

(c) If $U$ is an open subset of an lcs $E$, and $F$ is a semi-Montel space, then $H_{H Y}(U, F)$ is a semi-Montel space.

(d) Let $E$ be a metrizable lcs or the strong dual of a Fréchet-Montel space and $U$ an open subset of $E$. Then $H_{c o}(U)$ is semi-Montel.

Characterization of Schwartz spaces. We generalize a result in [1] to the case that we have a cvs $F$ as target space:

3. LeMMA. For a compact topological space $X$ and an $L_{c}$-embedded cvs $F, a$ net $\left(f_{i}\right)_{i}$ converges to zero in $C_{c}(X, F)$ if and only if it converges to zero uniformly on $X$ (with respect to the natural uniform convergence structure on $F$ ).

ProOF. Clearly a net, which converges uniformly, also converges in $C_{c}(X, F)$. Conversely, let $\left(f_{i}\right)_{i}$ converge to zero in $C_{c}(X, F)$ and assume that the convergence is not uniform on $X$. Since $F$ is embedded into $L_{c} L_{c} F$, there is a net $\left(l_{k}\right)_{k}$ in $L_{c} F$, which converges to some $l \in L F$, such that $l_{k} \circ f_{i}(x)$ does not converge to zero uniformly on $X$. Now there exist an $\varepsilon>0$, subnets $\left(l_{\kappa}\right)_{\kappa}$ and $\left(f_{\iota}\right)_{\iota}$ of the nets $\left(l_{k}\right)_{k}$ and $\left(f_{i}\right)_{i}$, respectively, and a net $\left(x_{\iota \kappa}\right)_{\iota \kappa}$ in $X$, such that $\left|l_{\kappa} \circ f_{\iota}\left(x_{\iota \kappa}\right)\right| \geq \varepsilon$ for every $\iota$ and $\kappa$. But since $\left(x_{\iota \kappa}\right)_{\iota \kappa}$ has a convergent subnet, this leads to the contradiction $0 \geq \varepsilon$. Hence $\left(f_{i}\right)_{i}$ converges to zero uniformly on $X$.

Next we prove a preparatory result, which will be phrased in terms of cvs although we shall not need it in its full generality in this paper. It must be stressed that it is essential for the proof of Theorem 6 , that this result can be obtained for arbitrary quasi-bounded nets in $H_{c}(U, F)$ and not only for bounded nets.

4. Proposition. Let $U$ be a circled, convex, and open subset of a cvs $E$, let $F$ be an $L_{\alpha}$-embedded cvs $(\alpha=c$ or $e)$, and let

$$
\gamma: H_{\alpha}(U, F) \rightarrow \prod_{m=0}^{\infty} P_{\alpha}\left({ }^{m} E, F\right) \quad(\alpha=c \text { or } e)
$$

be the continuous linear mapping defined by $\gamma(f)=\left(\hat{d}^{m} f(0)\right)_{m}$. If a net $\left(f_{i}\right)_{i}$ in $H_{\alpha}(U, F)$ is quasi-bounded and if the net $\left(\gamma\left(f_{i}\right)\right)_{i}$ converges to zero in $\prod_{m} P_{\alpha}\left({ }^{m} E, F\right)$, then $\left(f_{i}\right)_{i}$ converges to zero in $H_{\alpha}(U, F)(\alpha=c$ or $e)$.

ProOF. Let $\left(f_{i}\right)_{i}$ be a quasi-bounded net in $H_{\alpha}(U, F)$, such that $\hat{d}^{m} f_{i}(0)$ converges to 0 in $P_{\alpha}\left({ }^{m} E, F\right)$ for each $m=0,1, \ldots$ We shall prove that $f_{i} \rightarrow 0$ in $H_{\alpha}(U, F)$. Since the mapping $H_{\alpha}(U, F) \rightarrow C_{\alpha}\left(U \times L_{c} F\right), f \mapsto((x, l) \mapsto l \circ f(x))$, is an embedding, we have in the case $\alpha=c$ to prove that $l_{k} \circ f_{i}\left(h_{j}\right) \rightarrow 0$ for each 
net $\left(h_{j}, l_{k}\right)_{j k}$, which converges to $(h, l) \in U \times L_{c} F$. In the case that $\alpha=e$, we have to prove that there exist $j_{0}$ and $k_{0}$, such that for each $\varepsilon>0$ exists an $i_{0}$ with $\left|l_{k} \circ f_{i}\left(h_{j}\right)\right|<\varepsilon$ for $(i, j, k) \geq\left(i_{0}, j_{0}, k_{0}\right)$. According to [4]

$$
l_{k} \circ f_{i}\left(h_{j}\right)=\sum_{m=0}^{N} \frac{l_{k} \circ \hat{d}^{m} f_{i}(0) h_{j}}{m !}+l_{k} \circ R_{N}\left(f_{i}, h_{j}\right),
$$

where

$$
R_{N}\left(f_{i}, h_{j}\right)=\frac{1}{2 \pi i} \int_{|\lambda|=\rho>1} \frac{f_{i}\left(\lambda h_{j}\right)}{\lambda^{N+1}(\lambda-1)} d \lambda
$$

and where $\left(h_{j}, l_{k}\right) \rightarrow(h, l)$ in $U \times L_{c} F$. Let $\rho>1$ be such that $\rho h \in U$. For an arbitrary $\eta$ with $1<\eta<\rho$ we have a continuous mapping $L_{c} F \times H_{\alpha}(U, F) \times \bar{D}_{\eta} \times$ $\rho^{-1} U \rightarrow \mathbf{C},(l, f, \lambda, h) \mapsto l \circ f(\lambda h)$, where $\bar{D}_{\eta}=\{\lambda \in \mathbf{C}:|\lambda| \leq \eta\}$. The associated mapping $L_{c} F \times H_{\alpha}(U, F) \times \rho^{-1} U \rightarrow C_{c}\left(D_{\eta}\right)$ is continuous by the universal property of continuous convergence [1]. According to Lemma 3 the net $\left(\lambda \mapsto l_{k} \circ n^{-1} f_{i}\left(\lambda h_{j}\right)\right)$ converges to zero uniformly on $D_{\eta}$. Therefore, there are $i_{0}, j_{0}, k_{0}$, and $n_{0}$, such that $\sup _{|\lambda|=\eta}\left|l_{k} \circ f_{i}\left(\lambda h_{j}\right)\right| \leq n_{0}$ for $(i, j, k) \geq\left(i_{0}, j_{0}, k_{0}\right)$. Since

$$
\left|l_{k} \circ R_{N}\left(f_{i}, h_{j}\right)\right| \leq 1 / \eta^{N}(\eta-1) \sup _{|\lambda|=\eta}\left|l_{k} \circ f_{i}\left(\lambda h_{j}\right)\right|,
$$

there is, for a given $\varepsilon>0$, a number $N$, such that $\left|l_{k} \circ R_{N}\left(f_{i}, h_{j}\right)\right|<\varepsilon / 2$ for $(i, j, k) \geq\left(i_{0}, j_{0}, k_{0}\right)$ (with the case $\alpha=e$ in mind, observe that $i_{0}, j_{0}$, and $k_{0}$ do not depend on $\varepsilon)$. For each $m \in\{0,1, \ldots, N\}$ the net $\left(l_{k} \circ \hat{d}^{m} f_{i}(0)\right)_{i k}$ converges to zero in $P_{\alpha}\left({ }^{m} E, F\right)(\alpha=c$ or $e)$. Thus there is, in the case $\alpha=c$, a triple $\left(i_{1}, j_{1}, k_{1}\right)$ with $\left(i_{1}, j_{1}, k_{1}\right) \geq\left(i_{0}, j_{0}, k_{0}\right)$, such that

$$
\left|\sum_{m=0}^{N} \frac{l_{k} \circ \hat{d}^{m} f_{i}(0) h_{j}}{m !}\right|<\frac{\varepsilon}{2}
$$

so consequently $\left|l_{k} \circ f_{i}\left(h_{j}\right)\right|<\varepsilon$ for $(i, j, k) \geq\left(i_{1}, j_{1}, k_{1}\right)$. Hence $\left(f_{i}\right)_{i}$ converges to zero in $H_{c}(U, F)$. In the case $\alpha=e$, there clearly exist $j_{1}$ and $k_{1}$, such that for each $\varepsilon>0$ exists an $i_{1} \geq i_{0}$, such that (1) holds for $(i, j, k) \geq\left(i_{1}, j_{1}, k_{1}\right)$. Hence $\left(f_{i}\right)_{i}$ converges to zero in $H_{e}(U, F)$.

The local nature of the convergence structures $c$ and $e$ immediately gives

5. LEMMA. If $\left(U_{i}\right)_{i}$ is an open covering of an open subset $U$ of an lcs $E$, then the canonical mapping $H_{\alpha}(U) \rightarrow \prod_{i} H_{\alpha}\left(U_{i}\right)(\alpha=c$ or $e)$ is an embedding.

Now we can state

6. THEOREM. Let $U$ be an open subset of an lcs E. Then $E$ is a Schwartz space if and only if $H_{c}(U)=H_{e}(U)$.

PROOF. Because of Lemma 5 it suffices to prove the theorem for a circled convex $U$. If $H_{c}(U)=H_{e}(U)$ then $L_{c} E=L_{e} E$, and consequently $E$ is Schwartz [11]. Conversely, if $E$ is Schwartz, then $P_{c}\left({ }^{m} E\right)=P_{e}\left({ }^{m} E\right)$ for each $m \in \mathbf{N}$ (cf. [5, Proposition 2.3]). Consider an arbitrary net $\left(f_{i}\right)_{i}$, which converges to zero in $H_{c}(U)$. Then $\left(f_{i}\right)_{i}$ is quasi-bounded in $H_{c}(U)$ and $\left(\hat{d}^{m} f_{i}(0) / m !\right)_{i}$ converges to zero in $P_{c}\left({ }^{m} E\right)=P_{e}\left({ }^{m} E\right)$ for each $m=0,1, \ldots$ Since $\left(f_{i}\right)_{i}$ is quasi-bounded also 
in $H_{e}(U)$ it follows by Proposition 4 that $\left(f_{i}\right)_{i}$ converges to zero in $H_{e}(U)$. Thus $H_{c}(U)=H_{e}(U)$.

REMARK. If one could prove that $H_{c}(U)$ (or $H_{e}(U)$ ) is a Schwartz cvs [12] when $U$ is an open subset of a Schwartz lcs, then one would have an equality $H_{c}(U, F)=H_{e}(U, F)$ for any Schwartz lcs $F$.

6.1. COROLLARY. If $U$ is an open subset of a Schwartz lcs $E$, then all bounded sets in $H_{e}(U)$ are relatively compact in $H_{e}(U)$.

Every cvs $E$ defines an les $E_{\tau}$, which is the same vector space provided with the finest locally convex topology coarser than the convergence structure of $E$. It is known that $C_{c}(X)_{\tau}=C_{c o}(X)$, if $X$ is a $c$-embedded space (cf. [1, Theorem 37]). On the dual $L E$ of an lcs $E, L_{c o} \hat{E}=L_{c} E_{\tau}$ (cf. [10, Theorem 9.3.7]), where $\hat{E}$ denotes the completion of $E$. These two results suggest that a similar result could be valid for spaces of holomorphic functions. But the following example shows, that the situation is completely different in the holomorphic case.

EXAMPLE. Consider the topological product $E=\mathbf{C}^{\mathbf{N}}$. The space $E$ is a nuclear Fréchet space. Hence $H_{c o}(E)=H_{\tau_{\omega}}(E)$ and $H_{c o}(E) \neq H_{\tau_{\delta}}(E)$ (cf. Corollary 6.40 and example 2.52 in [7]). Since every nuclear Fréchet space is separable, $H_{e}(E)_{\tau}=H_{\tau_{\delta}}(E)$ by [4, Corollary 3.3]. But since $H_{c}(E)=H_{e}(E)$ by Theorem 6, we can conclude that $H_{c}(E)_{\tau}=H_{e}(E)_{\tau}=H_{\tau_{\delta}}(E) \neq H_{c o}(E)=H_{\tau_{\omega}}(E)$.

QUESTION. It would be interesting to know whether the equality $H_{c}(E)_{\tau}=$ $H_{c o}(E)$ holds or not for a Banach space $E$.

\section{REFERENCES}

1. E. Binz, Continuous convergence in $C(X)$, Lecture Notes in Math., vol. 469, Springer-Verlag, Berlin, Heidelberg and New York, 1975.

2. S. Bjon, Einbettbarkeit in den Bidualraum und Darstellbarkeit als projektiver Limes in Kategorien von Limesvektorräumen, Math. Nachr. 97 (1979), 103-116.

3. __ On an exponential law for spaces of holomorphic mappings, Math. Nachr. 131 (1987), 201-204.

4. S. Bjon and M. Lindström, A general approach to infinite-dimensional holomorphy, Monatsh. Math. 101 (1986), 11-26.

5. __ On a bornological structure in infinite-dimensional holomorphy, Math. Nachr. (to appear).

6. C. H. Cook and H. R. Fischer, On equicontinuity and continuous convergence, Math. Ann. 159 (1965), 94-104.

7. S. Dineen, Complex analysis in locally convex spaces, North-Holland Math. Stud., vol. 57, North-Holland, Amsterdam, New York, and Oxford, 1981.

8. A. Frölicher and W. Bucher, Calculus in vector spaces without norm, Lecture Notes in Math., vol. 30, Springer-Verlag, Berlin, Heidelberg and New York, 1966.

9. W. Gähler, Grundstrukturen der Analysis II, Akademie-Verlag, Berlin, 1978.

10. H. Jarchow, Locally convex spaces, Teubner, Stuttgart, 1981.

11. _ Duale Characterisierung der Schwartz-Räume, Math. Ann. 196 (1972), 85-90.

12. M. Lindström, On Schwartz convergence vector spaces, Math. Nachr. 117 (1984), 37-49.

Matematiska Institutionen, Ábo Akademi, Fänriksgatan 3, SF-20500 Åbo, FinLAND 